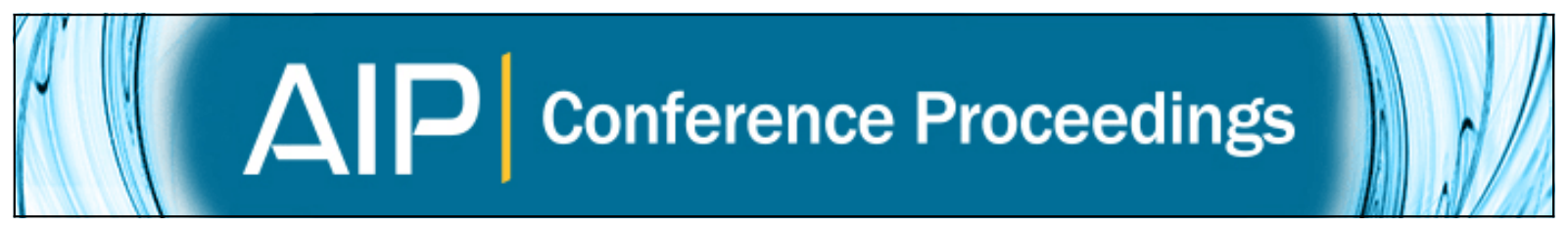

\title{
Symposium on Data Assimilation and Inverse Problems in Geosciences
}

Ibrahim Hoteit

Citation: AIP Conference Proceedings 1281, 1069 (2010); doi: 10.1063/1.3497820

View online: http://dx.doi.org/10.1063/1.3497820

View Table of Contents: http://scitation.aip.org/content/aip/proceeding/aipcp/1281?ver=pdfcov

Published by the AIP Publishing

Articles you may be interested in

Symposium: Recent developments in numerical schemes for Hilbert space related issues in science and engineering

AIP Conf. Proc. 1504, 784 (2012); 10.1063/1.4771810

Sequential Inverse Problems Bayesian Principles and the Logistic Map Example

AIP Conf. Proc. 1281, 1071 (2010); 10.1063/1.3497821

An inverse problem for Gibbs fields with hard core potential

J. Math. Phys. 48, 053301 (2007); 10.1063/1.2719141

Backscattering and inverse problem in random media

J. Math. Phys. 40, 4359 (1999); 10.1063/1.532972

Theory and examples of the inverse Frobenius-Perron problem for complete chaotic maps

Chaos 9, 357 (1999); 10.1063/1.166413 


\title{
Symposium on Data Assimilation and Inverse Problems in Geosciences
}

\author{
Ibrahim Hoteit \\ Applied Mathematics and Computer Sciences division, King Abdullah University of Sciences and Technology, KSA
}

PREFACE

Data assimilation and inverse methods combine dynamical models and observations to make the best possible estimates of the state and/or the parameters of a dynamical system. With the development of complex numerical models in almost every area of geosciences, these methods are increasingly becoming a necessary component to optimize the numerical models and to achieve more accurate predictions and reanalysis. The high complexity, nonlinearity and large dimension make the application of data assimilation and inverse methods to geophysical systems a quite challenging task.

While data assimilation and inverse problems have the same theoretical background and they are both approaches to tackle the same problem, assimilation and inverse methods were historically developed by two different communities; the first focuses on the estimation (can be prediction or analysis) of the system state, and the second on the estimation of the system parameters (generally with the goal of optimizing the system state). This resulted in the introduction of different approaches and algorithms specific to each community. The aim of this mini-symposium is to gather and discuss recent theoretical and practical progress relevant to data assimilation and inverse problems in all areas of geosciences. This includes, but is not limited to, applications in atmosphere, ocean, climate, solid earth geophysics, hydrology, and petroleum engineering. The mini-symposium will also discuss contributions discussing new developments of operational systems in meteorology, oceanography and hydrology. Special emphasis is put on methods and new developments of mathematical aspects of data assimilation and inverse problems including

- Statistical and deterministic approaches to inverse problems and data assimilation

- Inverse problems and data assimilation with very high dimensional parameter and state spaces

- Data assimilation methods, e.g. four-dimensional variational data assimilation (4DVAR), Kalman filtering and smoothing, Ensemble and particle filtering, and more generally nonlinear Bayesian filtering

Ensemble Kalman filtering (EnKF) methods are now widely used in all areas of geosciences. These ad-hoc methods combine the correction filter correction of the Kalman filter and the Monte Carlo forecast step of the particle filter. They are particularly appealing because they were proven efficient at reasonable computational burden, and because they are portable as their implementation do not require any change to the nonlinear system. EnKF methods are however subject to sampling errors because of the finite-size ensemble representation of the state probability distribution function (pdf). The work "On ensemble nonlinear Kalman filtering with symmetric analysis ensembles" discusses how the introduction of certain symmetry to the ensembles can reduce the sampling errors and spurious modes in evaluation of the means and covariances of the ensembles.

The articles "Particle Kalman filtering: A nonlinear framework for ensemble Kalman filters" and "Regularized Particle Filter with Langevin Resampling Step" discuss approaches (and approximations) to derive EnKF methods directly from the optimal Bayesian filter (instead of the linear Kalman filter). The first presents a discrete solution of the optimal nonlinear Bayesian filtering problem based on Gaussian mixture representation of the state probability distribution function. It is shown that this so-called particle Kalman filter generalizes the optimality of the analysis step of ensemble Kalman filtering methods to nonlinear systems. The second introduces a global resampling step to the regularized particle filter to derive the ensemble square root filter.

4DVAR methods are usually used to identify uncertain parameters in large-scale models. This however requires the implementation of the adjoint model, which requires an important programming effort. The work "Identification of Uncertain Parameters in a Tidal Model of the North Sea by SPSA" shows that the SPSA method, which determines the steepest directions of the inverse problem based on stochastic simultaneous perturbations, leads to comparable results to steepest descent method with little computational cost.

The article "Sequential Inverse Problems Bayesian Principles and the LogisticMap Example" discusses the parameters estimation problem in the presence of other sources of model uncertainties. It is argued that, when implementation problems are minimal, the Bayesian inversion framework is quite adequate.

CP1281, ICNAAM, Numerical Analysis and Applied Mathematics, International Conference 2010, Vol.II

edited by T. E. Simos, G. Psihoyios, and Ch. Tsitouras

(c) 2010 American Institute of Physics 978-0-7354-0835-7/10/\$30.00 
Other presentations (not included in the mini-symposium proceedings) discussed the large dimensional Bayesian inversion problem with application to a reservoir model (B. Mallick), the combination of two different statistical tools Kolmogorov/Zurbenko and Kalman filters - to eliminate model systematic errors (G. Kalanis and G. Kallos), the development of high-resolution EnKF-based assimilation system for the Mediterranean Sea (G. Korres et al.), the Effect of random perturbations on adaptive observation techniques (M.J. Hossen et al), comparison of the adjoint and forward forward sensitivity based approach to dynamic data assimilation (S. Lakshmivarahan), an ensemble implementation of the H-infinity filter with application to a coupled marine ecosystem model (G. Triantafyllou et al.), and an Overview of Multiscale Seismic Inversion with Phase-Encoded Sources (C. Boonyasiriwat).

\section{Dr. Ibrahim Hoteit}

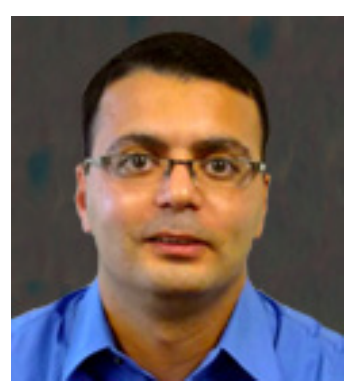

Dr. Ibrahim Hoteit was born in Beirut. He pursued his undergraduate and graduate studies in Mathematics at the Université Joseph Fourrier (UJF) in France. After obtaining his doctorate in Applied Mathematics in 2001 from UJF, he moved to the United States, initially to work as a postdoctoral researcher at Scripps Institution of Oceanography (SIO) University of California at San Diego - within the Estimating of the Circulation and the Climate of the Ocean (ECCO - MIT/SIO/JPL) consortium. Since his appointment in 2004, Dr. Hoteit has served as a research scientist at SIO.

Dr. Hoteit obtained his doctoral degree in Applied Mathematics working on the Kalman filter-based assimilation techniques for applications to high-dimensional nonlinear dynamical systems. At SIO, he was interested in the theoretical and practical development of data assimilation and inverse problems methods suitable for application to realistic numerical ocean models.

In December 2008, Dr. Hoteit was appointed an Assistant Professor in Applied Mathematics and Computational Science at King Abdullah University in Sciences and Technology (KAUST). 